\title{
The renin-angiotensin system and the heart: beyond 2000
}

\author{
T Morgan, H R Brunner
}

The renin-angiotensin system is an important controller of the circulation, but even if completely inhibited, circulatory control still persists. It was initially thought to be a blood borne system, in which renin released from the kidneys leads to the formation of angiotensin II in plasma, which causes vasoconstriction and the release of aldosterone from the adrenal glands. Since then, a multitude of other actions and controls has been discovered.

\section{Local production of renin and angiotensin II}

In the kidney, there is a release of renin ${ }^{1}$ and probably angiotensin $\mathrm{II}^{2}$ into the interstitium. Angiotensin II may work locally, altering proximal tubule reabsorption, ${ }^{3}$ afferent and efferent arteriole tone, and possibly glomerular mesangial and epithelial function. The intrarenal system operates constantly, independent of the systemic needs and often independently of the circulating angiotensin II and renin concentrations. It is important for maintenance and regulation of renal blood flow, glomerular filtration rate, glomerulotubular balance, and tubuloglomerular feedback, interacting with other locally produced and systemic hormones. The intrarenal role of the renin-angiotensin system may be of prime importance, with the release of renin into the circulation either a spillover phenomenon or a "fallback" system that works systematically in situations of hypotension or volume depletion.

Evidence for local production and action of angiotensin II has been obtained for a variety of tissues : blood vessels, ${ }^{5}$ heart, ${ }^{6}$ adrenals, ${ }^{7}$ and the brain, ${ }^{8}$ to mention the tissues most studied. Compared with the kidney, there is an important difference. The kidney releases renin into the circulation, but it is doubtful if under normal circumstances any of the other tissues studied make a significant contribution to active plasma renin. Messenger RNA for renin have been demonstrated by polymerase chain reaction (PCR) methodology, but it is unclear if in blood vessels and the heart this leads to renin synthesis. ${ }^{9}$ Danser and Schalekamp ${ }^{10}$ showed that under normal circumstances renin in the heart comes from the kidney through the plasma. Similar results probably apply to blood vessels. Thus in the heart and blood vessels, renin concentrations depend on plasma renin concentration (fig 1). The values in the different tissues are not identical and may follow a different time course, due to the presence of renin binding proteins in the membranes of these tissues. ${ }^{10}$ Still, the primacy of the kidney in controlling this component of the renin-angiotensin system must be recognised. Whether renin can also be produced locally by the heart in pathological situations has not been adequately established. The heart can form angiotensin I locally and convert this to angiotensin II, achieving levels two to three times higher than are present in plasma. ${ }^{10}$ The heart could regulate angiotensin II production by altering the amount of renin binding protein, the amount of converting enzyme, or the amount of angiotensinogen produced. Local actions depend upon the presence of relevant angiotensin receptors, which then alter the composition of the cell, leading to physiological or pathological actions. There are two receptor subtypes for angiotensin II. The role of the $\mathrm{AT}_{2}$ receptor is unknown, though it has been postulated that it may have an antihypertrophic effect. ${ }^{11}$ The $A T_{1}$ receptor has been clearly identified and is associated with vasoconstriction, ${ }^{12}$ renal sodium absorption, ${ }^{13}$ inhibition of renin release, ${ }^{14}$ aldosterone release, ${ }^{15}$ and cell growth. ${ }^{12}$ Thus the balance of the $\mathrm{AT}_{1}$ and $\mathrm{AT}_{2}$ receptor subtypes would determine whether or not hypertrophy occurs. This is a proposition that needs to be tested.

\section{Local effects of angiotensin II: the important role of multiple interacting factors}

Angiotensin II produced in the heart has a number of actions (fig 1). Working through the $\mathrm{AT}_{1}$ receptor, it can cause local regulation of vascular tone and blood flow, and increases in cardiocyte contractility. ${ }^{16}$ It has been shown in vitro that it is a potent growth factor, increasing the production of a variety of proteins that are related to cardiac hypertrophy. ${ }^{17}$ Yamazaki et $a l^{18}$ have shown in vitro that mechanical stretch of cardiocytes activates protein kinases, which would cause translocation of the signal into the nucleus. Furthermore, the presence of an $\mathrm{AT}_{1}$ receptor blocking drug prevents in part the activation of this cascade. Angiotensin II is released in vitro from these stretched cardiocytes, though this was not found consistently. This could provide evidence for the involvement of angiotensin II as a local autocrine or paracrine system involved with cardiocyte hypertrophy. In contrast, the importance of plasma renin in vivo must be emphasised, as this will regulate the amount of cardiac renin, and thus angiotensin I production. 


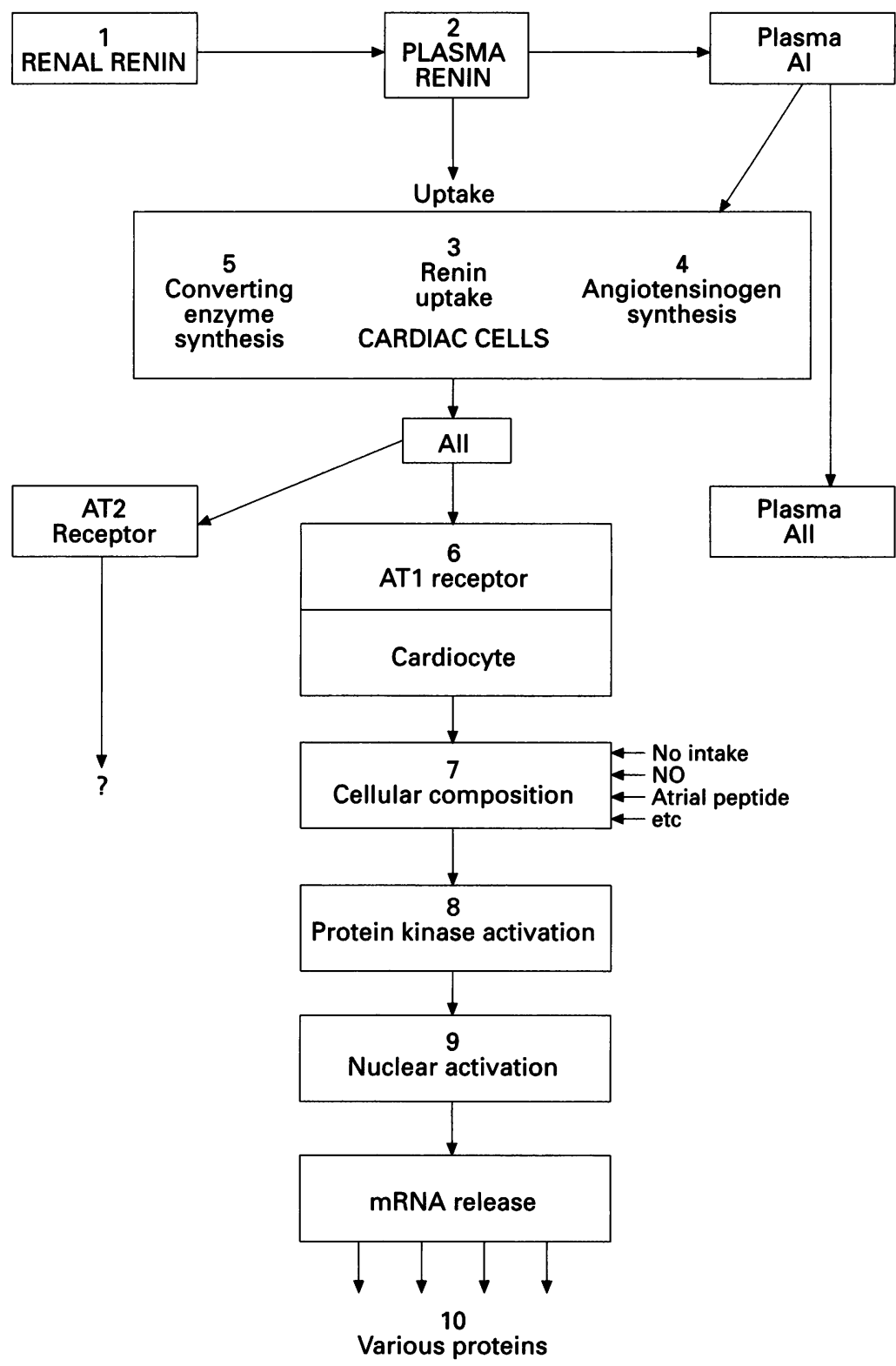

Figure 1 An outline of the action of the RAS in the heart, indicating its interaction with the plasma system and sites where there are interactions with other systems. Sites where the process may be interrupted are indicated: (1) reduce renal renin secretion; (2) inhibit plasma renin; (3) prevent cardiac uptake of renin; (4) reduce cardiac angiotensinogen production; (5) reduce cardiac converting enzyme production or activity; (6) prevent action of angiotensin II by $A T_{1}$ receptor blocking drugs; (7) modify effect of angiotensin II on cellular composition; (8) modify activation of protein kinase cascade; (9) prevent nuclear activation of specific DNA; (10) prevent expression of end products that may be harmful. breakdown of bradykinin. These results have not been confirmed in the same model ${ }^{24}$ and have also not been observed in other models of hypertension and cardiac hypertrophy. ${ }^{25}$ In a model of cardiac hypertrophy due to aortocaval fistula, two groups ${ }^{26-28}$ showed that blockade of the renin-angiotensin system with losartan, quinapril, or lisinopril caused resolution of both right and left ventricular hypertrophy. Even here, there was a discrepancy, as enalapril did not cause resolution. This was ascribed to a lower specificity of enalapril for cardiac converting enzyme, based on a paper by Cushman et al. ${ }^{29}$ However, the conclusions in that paper are open to several different interpretations, as it was an acute single dose study. Also, ramipril was as ineffective as enalapril at inhibiting cardiac ACE, whereas in papers quoted above, ${ }^{22} 23$ it was claimed to be particularly effective at reducing left ventricular hypertrophy.

Angiotensin II acting locally is probably important in left ventricular hypertrophy, but the exact relation between workload, wall stress, angiotensin II, aldosterone, atrial natriuretic factor, sodium intake, nitric oxide, and a multitude of other variables needs careful study and documentation. Thus, is it possible to cause left ventricular hypertrophy in the complete absence of angiotensin II, or can other factors have similar effects? Can angiotensin II induce left ventricular hypertrophy in the complete absence of enhanced wall stress? Until these data are available, it is difficult, if not impossible, to interpret the effects of angiotensin II on protein kinases, or production of different mRNA. The effects are present but do they cause hypertrophy?

Angiotensin II exerts its major effects by binding to an $\mathrm{AT}_{1}$ receptor and thereby probably alters the cell sodium concentration. This in turn alters cytosolic calcium and activates the protein kinase cascade, which then transduces the signal into the nucleus, activating the processes concerned with hypertrophy. It is important to recognise that it is the alteration in cell composition that mediates the effect, rather than the direct angiotensin II concentration. Thus a high angiotensin II level in the absence of receptors may have little effect, while a low angiotensin II level with increased number of high affinity receptors may have an effect. Other factors may also modulate the cellular composition. Thus a high sodium diet may cause increased cell sodium levels with alterations in cytosolic calcium kinetics; nitric acid may alter cytosolic calcium; calcium channel blocking drugs may alter cytosolic calcium. So the effect of angiotensin II in vivo cannot be considered in isolation.

The multiple interactions between angiotensin II and sodium intake are of particular relevance. High sodium intake ${ }^{30}$ and high angiotensin $\mathrm{II}^{20}$ can cause cardiac hypertrophy and vascular stiffness. Low sodium intake reduces blood pressure and cardiac hypertrophy, but at the same time angiotensin II levels rise. Thus angiotensin II is not of isolated fundamental importance, but rather it reflects the sure but inhibit cardiac ACE cause regression of left ventricular hypertrophy in the aortic coarctation model, probably by preventing 
interaction of sodium balance and angiotensin II affecting cellular composition and triggering the cascade of events. A similar situation applies to the genesis of hypertension in the spontaneously hypertensive rat (SHR). Normalisation of blood pressure with an ACE inhibitor or an $\mathrm{AT}_{1}$ receptor blocking drug, but not with other drugs, attenuates chronically the blood pressure rise that develops when medication is stopped. ${ }^{3132}$ Likewise, treatment of an SHR during pregnancy or of the offspring for the first six weeks of life with a low salt intake chronically attenuates the blood pressure that develops when rats return to a normal sodium diet. ${ }^{334}$ Both treatments improve survival. Once again, the low sodium diet would cause high angiotensin II concentrations, but under these circumstances there are no adverse effects. This emphasises the question of appropriateness of the angiotensin II concentrations in relation to the sodium balance of a person or animal. Similar interactions affecting cellular composition may also exist with atrial natriuretic factor, nitric oxide, endothelin, and other factors that can alter cell composition.

Cardiac hypertrophy, coronary atherosclerosis, and eventually cardiac death are intimately intertwined with the renin-angiotensin system either in the plasma, or more specifically, in the heart where the local effects are regulated, in part, by the plasma renin concentration. The hope is that by specific modulation of the cardiac action of the various components, we can significantly alter the rate of development, the rate of progression, and the resolution of these disease processes.

\section{Genes of the renin-angiotensin system as markers of cardiovascular risk}

A major search is on to identify genetic markers that will enable us to detect individuals at risk of hypertension or its complications, enabling us to target appropriate people to treat with lifestyle modifications or drugs at an early age. Harrap ${ }^{35}$ reviews the evidence related to this and emphasises the need to test hypotheses rather than just generate them. The idea is that the genomic nature of a person will predict who is at risk of developing complications and problems. This aim is too ambitious and is unlikely to be achieved, though it will contribute to understanding and knowledge of these processes. The present data provide evidence of linkage of a genetic variant to a disease process. Occasionally a direct mechanistic model has evolved and more of these may exist. ${ }^{36}$ However, genetic makeup is unlikely to be all important without taking into account the multiple interactions with various environmental factors. In a hunter-gatherer community there is little or no hypertension, no atherosclerosis and little cardiac hypertrophy. The genetic propensity for all these is present but is not expressed in that environment. If the environment alters, the genetic makeup may determine who will develop these problems.

If overactivation of the renin-angiotensin system in the heart is associated with adverse cardiac effects, it would appear that the critical measurement is the angiotensin II level, the $\mathrm{AT}_{1}$ receptor level, and the resultant effect on cell composition. To examine, or know, the genotype that might control the activation or amount of converting enzyme (and thus angiotensin II) present in the heart appears more indirect than knowing the amount of angiotensin II.

We shall try to illustrate the complexity of the situation with the example of myocardial infarction (fig 2). The best "predictor" of a myocardial infarction is after it has occurred $(100 \%)$. The next best predictor is probably severe coronary atherosclerosis, but this is difficult to detect without invasive procedures. At the next level, we have plasma cholesterol (with different predictability of various subtypes), smoking, glucose intolerance, and raised blood pressure. These interact with each other but are all less predictive than the presence of atherosclerosis. Beneath these we have a balance of humoral factors that are genetically determined but also are controlled by the environment. Beneath these again we have the proteins produced according to the genetic process, and their production may be regulated by the genomic subtype influenced by a variety of environmental factors. Finally, at the bottom, we have the normal genome or variant that controls production. It is difficult to see how a genomic variant that has some effect on cholesterol synthesis would be a better predictor than cholesterol itself, unless it has a direct potent influence by some other mechanism on a cell process associated with cardiac hypertrophy, atherosclerosis, or sudden death. This appears unlikely as the hunter-gatherer does not have these problems. Thus the association of the genomic subtype with problems may be environmentally determined and may differ across environments. ${ }^{35}$

Even high up on the scale (fig 2), we have all observed patients with high cholesterol, high blood pressure, high renin, and heavy cigarette smoking who, at the age of 80 , have (still) developed no problems. Thus the possibility of "beneficial" genotypes needs to be considered. There are also important interactions between the genotypes. A patient may have an ACE DD genotype which may favour conversion of angiotensin I to angiotensin II, ${ }^{37}$ but they might also have an $\mathrm{AT}_{1}$ receptor gene subtype causing "less active" receptors. Thus there may be no net cellular effect. In contrast, they may have an ACE DD genotype and a variant of the $\mathrm{AT}_{1}$ receptor gene causing more active receptors, thereby increasing the effect of both. The combination of the ACE DD genotype and the $\mathrm{CC}$ variant of the $\mathrm{AT}_{1}$ receptor gene was more closely associated with myocardial infarction than either alone. ${ }^{38}$ The mechanism of this association is unknown.

There is little doubt that markers will be found that identify groups of people at risk, but in the individual patient they may not be as predictive as, for example, the cholesterol or its subtype levels. However, in the future there may be a greater number of such markers that can be readily determined early in life before 


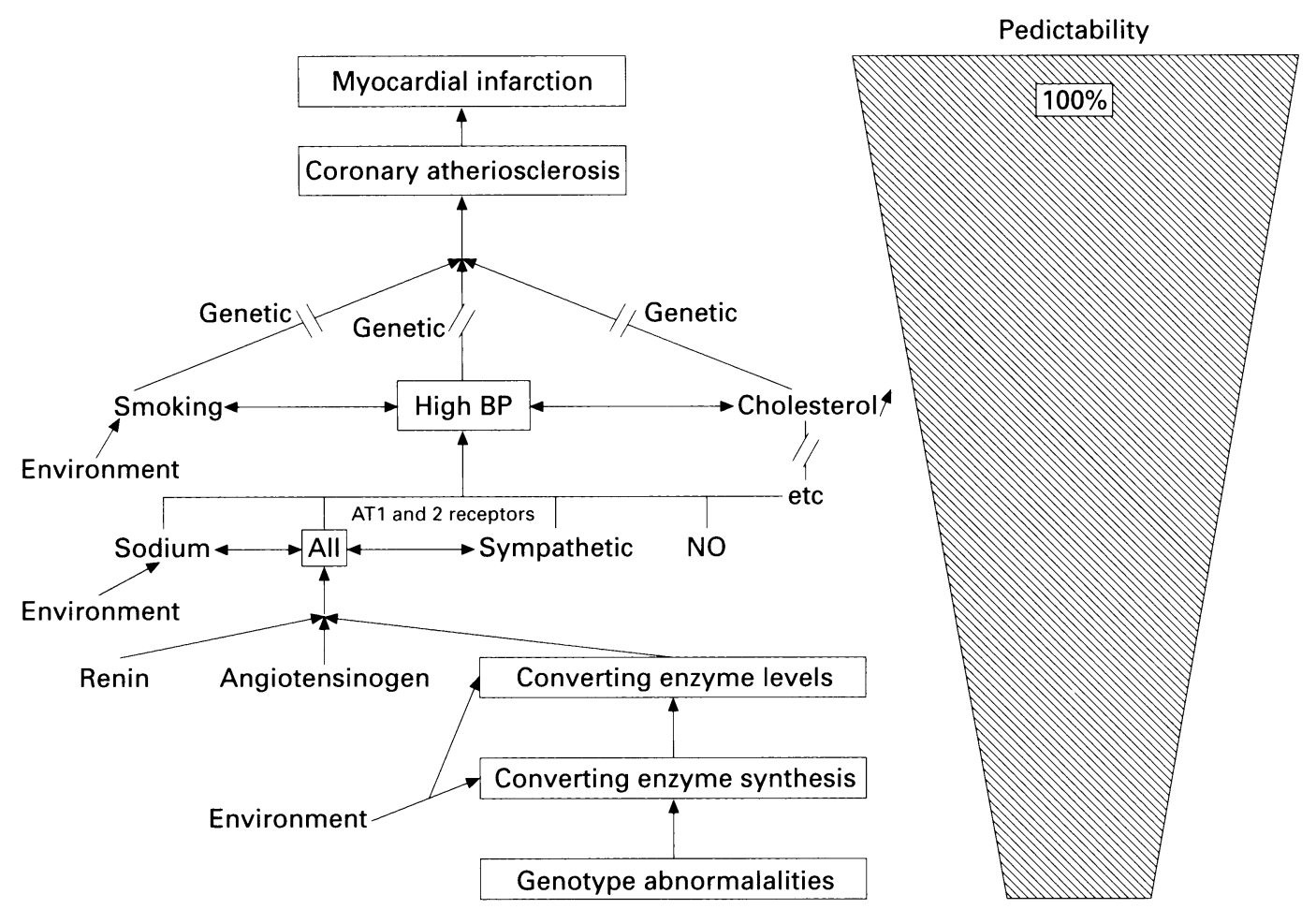

Figure 2 This is a simplified version of the antecedents of myocardial infarction. As one goes further away from the disease process itself the power of predictability becomes less and less. Thus to identify a genetic marker that works through a known pathway is unlikely to have as good a predictability as the end product of that genotype. However, it is feasible that a genotype may interfere with a process at a higher level (eg, cell proliferation) by mechanisms at present unknown, and thereby confer greater predictability than our present markers (see text). The values of predictability given are estimates only, and this schematic covers only a few of the interactions (eg, atrial natriuretic factor, aldosterone etc, are not shown). These interactions lead to greater complexity and to a reduced likelihood of a specific genotype acting through a known pathway being of overriding importance. Some other possible genetic effects are indicated, eg, why is smoking more harmful in some people? Is this genetically determined? These controls may become the powerful predictors.

they are expressed as a phenotype change. A constellation of such adverse markers, and the presence or absence of beneficial markers, may enable us to predict, treat, and prevent hypertension and cardiac disease at a very early stage. However, at present, the individual associations are weak, with large overlap between affected and unaffected people. ${ }^{39}$

\section{Blockade of the renin-angiotensin system} for more effective treatment

Treatment at present is pragmatic. Blockade of the renin-angiotensin system in the circulation, and also possibly locally, has improved prognosis in cardiac failure, ${ }^{40}$ and after myocardial infarction. ${ }^{4142}$ For hypertension, and possibly cardiac hypertrophy, it still remains to be proven that blockade of the renin-angiotensin system provides a better prognosis than blood pressure reduction per se. The mechanism of the improvement in cardiac failure and after myocardial infarction is obscure, and it is unclear if it is due to haemodynamic alterations, specific myocardial effects of the drugs, or - most likely - a combination of the two. The effect of ACE inhibitors may be mediated in part through the inhibition of bradykinin breakdown, leading to increased nitric oxide production and prevention of harmful effects. It is likely, from animal studies, that angiotensin II receptor blocking drugs have similar beneficial effects. An important long term therapeutic question is whether in the treatment of hypertension, the $\mathrm{ACE}$ inhibitors, the $\mathrm{AT}_{1}$ receptor blocking drugs, and the renin inhibitors have additional beneficial cardiovascular effects over and above those due to lowering blood pressure. Will they, if used early, prevent the development of atherosclerosis, as suggested by animal studies? Will they prevent, or cause regression of, cardiac hypertrophy better than other drugs? Finally, will they return the prognosis of the treated hypertensive patient back to that of a normotensive individual?

The future holds the possibility of identifying precisely where the deficit, or excessive action, exists. The genetic approach may allow us to provide preventative treatment before phenotype expression has developed. The cardiac renin-angiotensin system may be inactivated in a variety of ways. The development of a renin inhibitor may prevent the accumulation of renin in the heart, which is important in the cascade leading to angiotensin II effects. The membrane protein that binds renin may be identified, and competitive antagonists could be developed that target this protein and thereby prevent the uptake of renin from plasma, leading to specific cardiac depletion. Converting enzyme inhibitors may be developed that are specific for cardiac ACE (though this seems unlikely). Specific angiotensin II receptor blocking drugs will enable us to separate more clearly the role of angiotensin II reduction from bradykinin excess which occurs with ACE inhibitors. The role of the 
$\mathrm{AT}_{2}$ receptor and its relation to growth and hypertrophy needs to be established, but could lead to a different therapeutic approach. The above will reduce the effect of angiotensin II, but in addition, other substances and hormones that alter cellular composition need to be targeted. Thus to prevent adverse angiotensin II actions we may manipulate the cellular composition. Finally, the signal to the nucleus may be altered or interrupted, or the expression of DNA altered, in such a way that products that are beneficial will be produced by the cell rather than ones that are harmful. Cardiac cell growth and cardiocyte hypertrophy, when invoked by physiological processes, are beneficial. It is likely that properly coordinated cell growth and hypertrophy in cardiac failure, cardiac arrhythmia, and after myocardial infarction could also be beneficial. The challenge is to determine how this can be done.

\section{Summary}

Renin-angiotensin is both a circulatory and a local tissue system. However, in most circumstances, renin present in the heart and blood vessels is taken up from the plasma, and the kidney is the prime source of this renin. Tissues can then modulate and control the production of angiotensin II. In various organs, angiotensin II has local actions. In the heart, working through the $\mathrm{AT}_{1}$ receptor, it increases contractility and may cause cardiocyte hypertrophy. Indirectly, the heart is also very much affected by the vascular actions of angiotensin II. However, the net result on the heart is the product of an important interaction between a large number of factors. There is little doubt that an inappropriately high plasma (and tissue) level of renin, related to sodium balance, is associated with increased left ventricular hypertrophy and cardiovascular complications.

The genetic approach will lead to an understanding of genomic risk factors in hypertension and may identify control systems-at present unknown-which will lead to a new approach to the prevention of cardiovascular death. It is, however, unlikely that such a factor will clearly predict an individual's prognosis, but it will, rather, identify groups at risk. Constellations of genomic interactions with multiple environmental factors reduce this power.

Modification of the response to angiotensin II using present treatment, or interrupting the response at more distal sites, may enable us to achieve the beneficial effects on cardiac hypertrophy without the detrimental effects.

1 Morgan T, Davis JM. Renin secretion at the individual nephron level. Pflugers Arch 1975;359:23-31.

2 Mendelsohn FA. A method for measurement of angiorensin II in tissues and its application to rat kidney. Clin Sci Mol Med 1976;51:111-25.

3 Harris PJ, Young JA. Dose-dependent stimulation and inhibition of proximal tubular sodium reabsorption by
angiotensin II in the rat kidney. Pflugers Arch 1977;367: 295-7.

4 Campbell DJ, Kladis A, Duncan AM. Nephrectomy, converting enzyme inhibition, and angiotensin peptides. Hypertension 1993;22:513-22.
5 Dzau VJ, Gibbons GH, Cooke JP, Omoigui N. Vascular biology and medicine in the 1990s: scope, concepts, potentials, and perspectives. Circulation 1993;87:705-19.

6 Lindpaintner $\mathrm{K}$, Ganten $\mathrm{D}$. The cardiac renin angiotensin system. An appraisal of present experimental and clinical evidence. Circ Res 1991;68:905-21.

Naruse $M$, Takii $Y$, Inagami $T$. Renin exists in high concentration in the adrenal gland of the rat. Biomed Research $1981 ; 2: 583-4$

8 Ganten D, Hermann K, Bayer C, Unger T, Lang RE Angiotensin synthesis in the brain and increased turnove in hypertensive rats. Science 1982;222:869-71.

9 von Lutterotti N, Catanzaro DF, Sealey JE, Laragh JH. Renin is not synthesized by cardiac and extrarenal vascuar tissues. A review of experimental evidence. Circulation 1994;89:458-70.

10 Danser AHJ, Schalekamp MADH. Is there an internal cardiac renin angiotensin system? Heart 1996;76(suppl 3): 28-32 (this issue).

11 Stoll M, Steckelings UM, Paul M, Bottari SP, Metzger R, Unger $T$. The angiotensin AT2-receptor mediates inhibition of cell proliferation in coronary endothelial cells. $\mathcal{f}$ Clin Invest 1995;95:651-7.

12 Chiu AT, Roscoe WA, McCall DE Timmermans PB. Angiotensin II-1 receptor mediates both vasocontriction Angiotensin II-1 receptor mediates both vasocontriction cells. Receptor 1991;1:33-40.

13 Harris PJ, Hiranyachattada S, Antoine AM, Walker L Reilly AM, Eitle E. Regulation of renal tubular sodium ransport by angiotensin II and atrial natriuretic factor. Clin Exp Pharmacol Physiol (in press).

14 Zhang Y, Morgan T. Effects of enalapril and Dup753 on renin synthesis in mice with one hydronephrotic kidney. Clin Exp Pharmacol Physiol 1994;21:133-5.

15 Gupta P, Franco-Saenz R, Mulrow PJ. Locally generated angiotensin II in the adrenal gland regulates basal, corticotropin-, and potassium-stimulated aldosterone secretion. Hypertension 1995;25:443-8.

16 Ishihata A, Endoh M. Species-related differences in inotropic effects of angiotensin II in mammalian ventricuar muscle: receptors, subtypes and phosphoinositide hydrolysis. Br ₹ Pharmacol 1995;114:447-53.

17 Miyata S, Haneda T. Hypertrophic growth of cultured neonatal rat heart cells mediated by type 1 angiotensin II receptor. Am $\mathcal{F}$ Physiol 1994;266:H2443-51.

18 Yamazaki T, Komuro I, Shiojimo I, Yazaki Y. The reninangiotensin system and cardiac hypertrophy. Heart 1996;76 (suppl 3):33-5 (this issue).

19 Griffin SA, Brown WC, MacPherson F, McGrath JC, Wilson VG, Korsgaard N, et al. Angiotensin II causes vascular hypertrophy in part by a non-pressor mechavascular hypertrophy in part by a
nism. Hypertension 1991;17:626-35.

20 Dostal DE, Baker KM. Angiotensin II stimulation of lef ventricular hypertrophy in adult rat heart. Mediation by the AT1 receptor. Am $\mathcal{F}$ Hypertens 1992;5:276-80.

21 Kim S, Ohta K, Hamaguchi A, Yukimura T, Miura $K$ Iwao $\mathrm{H}$. Angiotensin II induces cardiac phenotypic modulation and remodeling in vivo in rats. Hypertension 1995;25:1252-9.

22 Linz W, Schölkens BA, Ganten D. Converting enzyme inhibition specifically prevents the development and induces regression of cardiac hypertrophy in rats. Clin Exp Hypertens (Theor Pract) 1989;A11:1325-50.

23 Linz W, Schaper J, Wiemer G, Albus U, Schölkens BA Ramipril prevents left ventricular hypertrophy with myocardial fibrosis without blood pressure reduction: one year study in rats. Br f Pharmacol 1992;107:970-5.

24 Rhaleb NE, Yang XP, Scicli AG, Carretero OA. Role of kinins and nitric oxide in the antihypertrophic effect of ramipril. Hypertension 1994;23:865-8.

25 Gohlke P, Linz W, Schölkens BA, Kuwer I, Bartenbach S, Schnell A, et al. Angiotensin-converting enzyme inhibition improves cardiac function. Role of bradykinin. tion improves cardiac fun

26 Ruzicka M, Yuan B, Harmsen E, Leenen FH. The reninangiotensin system and volume overload-induced cardiac angiotensin system and volume overload-induced cardiac
hypertrophy in rats. Effects of angiotensin converting hypertrophy in rats. Effects of angiotensin converting enzyme inhibitor versus angiot

27 Ruzicka M, Leenen FH. Relevance of blockade of cardiac and circulatory angiotensin-converting enzyme for the prevention of volume overload-induced cardiac hypertrophy. Circulation 1995;91:16-19.

28 Iwai N, Shimoike H, Kinoshita M. Cardiac reninangiotensin system in the hypertrophied heart. Circulation 1995;92:2690-6.

29 Cushman DW, Wang FL, Fung WC, Harvey CM DeForrest JM. Differentiation of angiotensin-convertin enzyme (ACE) inhibitors by their selective inhibition of ACE in physiologically important target organs. $A m \mathcal{F}$ Hypertens 1989;2:294-306.

30 Yuan BX, Leenen FH. Dietary sodium intake and left ventricular hypertrophy in normotensive rats. $A m \mathcal{F}$ Physiol 1991;261:H1397-401.

31 Harrap SB, Nicolaci JA, Doyle AE. Persistent effects on blood pressure and renal haemodynamics followin chronic angiotensin converting enzyme inhibition with perindopril. Clin Exp Pharmacol Physiol 1986;13:753-65.

32 Harrap SB, Van der Merwe WM, Griffin SA, Macpherso $\mathrm{F}$, Lever AF. Brief angiotensin converting enzym inhibitor treatment in young spontaneously hypertensive rats reduces blood pressure long-term. Hypertension 1990;16:603-14.

33 Di Nicolantonio R, Spargo S, Morgan TO. Prenatal high 
salt diet increases blood pressure and salt retention in teh spontaneously hypertensive rat. Clin Exp Pharmacol Physiol 1987;14:233-5.

34 Di Nicolantonio R, Silvapulle MJ, Spargo MJ, Morgan TO. High salt diet decreases longevity in the spontaneously hypertensive rat. Clin Exp Pharmacol Physiol neously hyperten

35 Harrap SB. Cardiovascular disease and genetics of the renin-angiotensin system. Heart 1996;76(suppl 3):

36 Dluhy RG, Lifton RP. Glucocorticoid-remediable aldosteronism. Endocrinol Metab Clin North Am 1994;23. 285-97.

37 Ueda S, Morton JJ, Elliot HL, Connell JMC. Enhanced pressor response to angiotensin I in normotensive males with DD type of angiotensin converting enzyme gene. $f$ Hypertens 1994;12:1324.

38 Tiret L, Bonnardeaux A, Poirier O, Ricard S, MarquesVidal P, Evans A, et al. Synergistic effects of angiotensinconverting enzyme and angiotensin-II type 1 receptor gene polymorphisms on risk of myocardial infarction. Lancet 1994;344:910-3.

39 Cambien F, Poirier O, Lecerf L, Evans A, Cambon JP, Arveiler D, et al. Deletion polymorphism in the gene for angiotensin converting enzyme is a potent risk factor for myocardial infarction. Nature 1992;359:641-44.

40 The SOLVD Investigators. Effect of enalapril on survival in patients with reduced left ventricular ejection fractions and congestive heart failure. $N$ Engl $f$ Med 1991;325: 293-302.

41 Pfeffer MA, Braunwald E, Moyé LA, Basta L, Brown EJ, Cuddy TE, et al. The SAVE Investigators. Effect of captopril on mortality and morbidity in patients with left ventricular dysfunction after myocardial infarction. Results of the survival and ventricular enlargement trial. N Engl f Med 1992;327:669-77.

42 The Acute Infarction Ramipril Efficacy (AIRE) Study Investigators. Effect of ramipril on mortality and morbidity of survivors of acute myocardial infarction with clinica evidence of heart failure. Lancet 1993;342:821-8. 